Universidad de Lima

Facultad de Psicología

Carrera de Psicología

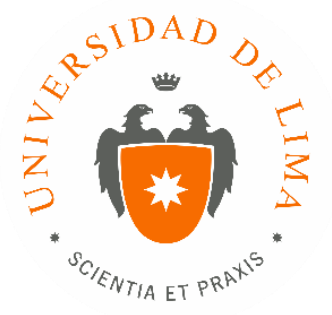

\title{
EXPERIENCIA DE TRABAJO EN UN HOSPITAL ONCOLÓGICO DE LIMA
}

Trabajo de suficiencia profesional para optar el título profesional de Licenciado en Psicología

\section{Sandra Daniela Ballón Grados}

Código 20102200

$$
\text { Lima - Perú }
$$

Febrero de 2019 


\section{EXPERIENCIA DE TRABAJO EN UN HOSPITAL ONCOLÓGICO DE LIMA}




\section{TABLA DE CONTENIDO}

INTRODUCCIÓN

CAPÍTULO 1: IDENTIFICACIÓN DEL PROBLEMA....................................................... 7

CAPÍTULO 2: DESCRIPCIÓN DE LAS ACTIVIDADES REALIZADAS.....................11

2.1 Acompañamiento psicológico a pacientes y familiares....................................11

2.1.1 Área de cirugía....................................................................... 12

2.1.2 Área de pediatría.....................................................................13

2.1.3 Área de adolescentes...............................................................13

2.1.4 Área de Unidad de Tratamiento Multidisciplinario (UTM), Unidad de Tratamiento Intermedio (UTI) y Unidad de Cuidados Intensivos (UCI).........14

2.2 Realización de talleres psicológicos.............................................................. 14

2.3 Orientación y consejería psicológica por consulta externa...............................16

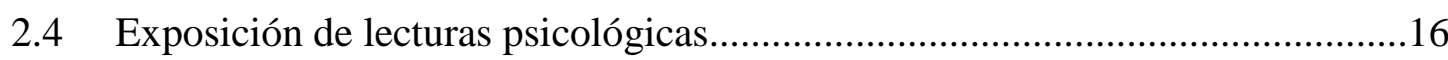

2.5 Supervisión de casos.................................................................................. 16

CAPÍTULO 3: RESULTADOS DE LA INTERVENCIÓN...................................................18

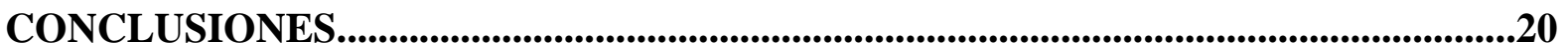

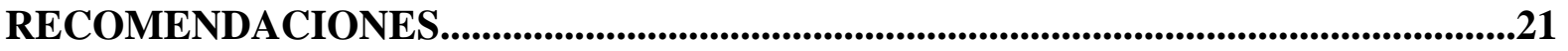

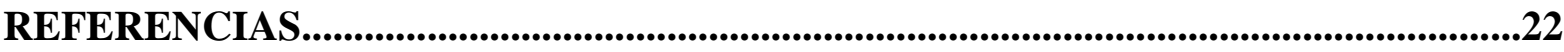

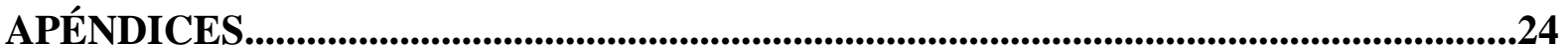




\section{ÍNDICE DE APÉNDICES}

Apéndice 1: Modelo de Nota Psicológica ............................................................................25

Apéndice 2: Formato de Taller Psicológico................................................................................26

Apéndice 3: Modelo de Supervisión de Caso......................................................................27 


\section{INTRODUCCIÓN}

Para la Organización Mundial de la Salud (OMS, 2018), el cáncer es un problema de salud pública, ya que es considerada la segunda causa de muerte a nivel mundial. En el contexto peruano, el Ministerio de Salud del Perú (MINSA, 2014) indicó que esta enfermedad ocupó el primer lugar en las causas de mortalidad por sexo. A pesar de los avances científicos y de la difusión pública acerca de ello, el cáncer aún es considerado como un prototipo de incurabilidad, sufrimiento, incapacidad y muerte (Cruzado, 2010). Es así que en el transcurso de la enfermedad y por diversos factores, los cuales serán mencionados más adelante, pueden desencadenarse sentimientos de ansiedad y depresión (Asociación Americana de Cáncer, 2016). Por ello, es fundamental el rol del psicólogo dentro de este contexto, ya que se debe intervenir tanto al paciente como a la familia e incluso, al personal sanitario (Cabrera, López, López y Arredondo, 2017).

El Instituto Nacional de Enfermedades Neoplásicas (INEN), uno de los pocos hospitales especializados en cáncer dentro del Perú, comienza a resaltar el apoyo psicólogico a pacientes y familias, realizando grupos de apoyo y dinámicas (INEN, 2017). Hoy en día, el departamento psicológico está conformado por ocho psicólogos y un grupo de aproximadamente veinte internos, los cuales tienen como objetivo principal brindar atención psicológica a pacientes y familiares de los tres grupos poblacionales dentro del hospital (niños, adolescentes y adultos) y, al mismo tiempo, brindar apoyo al personal asistencial. El presente trabajo tiene como finalidad describir las actividades realizadas por los internos, así como los resultados y logros obtenidos por las mismas. Finalmente, se mencionarán algunas recomendaciones que pueden servir como oportunidad de mejora, tanto para los miembros del programa de internado como para la misma institución. 


\section{CAPÍTULO I: IDENTIFICACIÓN DEL PROBLEMA}

En la actualidad, a pesar de los avances en los tratamientos de distintos tipos de tumores y de la difusión pública que se realiza de ello, el cáncer continúa siendo la enfermedad más temida, incluso más que las enfermedades cardiovasculares, debido a que es considerada como un prototipo de incurabilidad, sufrimiento, incapacidad y muerte, menciona Cruzado (2010).

De acuerdo con la Organización Mundial de la Salud (OMS, 2018), el cáncer es un problema de salud pública, siendo la segunda causa de muerte a nivel mundial; por ejemplo, en el año 2015 esta enfermedad ocasionó 8,8 millones de defunciones. Esta misma institución estima que para el año 2030, 21.3 millones de personas desarrollarán la enfermedad y 13.1 millones de personas morirán a causa de ella.

Según el Instituto Nacional de Enfermedades Neoplásicas (www.inen.sld.pe), en Latinoamérica el cáncer ocupa el tercer lugar de las causas de muerte. En el Perú, el Ministerio de Salud del Perú (MINSA, 2014) indicó que los tumores o neoplasias malignas ocuparon el primer lugar dentro de las principales causas de mortalidad por sexo. En el mismo año, el INEN (2014) registró 11,583 casos nuevos de cáncer en ambos sexos, siendo los de mayor incidencia: cáncer de cérvix, de mama, de estómago, de próstata y linfoma no hodking. Dicha cifra ha aumentado considerablemente desde el año 2000.

Salaverry (2013) sostiene que el 30\% de las causas de muerte por cáncer se dan por diversos factores de riesgo, tales como consumo de alcohol y tabaco, mala alimentación, obesidad, falta de actividad física, entre otras. Además, la misma autora plantea que el $70 \%$ de muertes ocasionadas por el cáncer, se dan en países de bajos y medianos ingresos, ya que destinan una menor inversión a la prevención y tratamiento de dicha enfermedad. 
A raíz de lo mencionado, Cruzado (2010) sostiene que la primera reacción ante el diagnóstico puede ser de negación, shock emocional o incredulidad. De igual manera, Fernández, Jorge, Sánchez y Bejar (2016) postulan que el diagnóstico de cáncer está fuertemente vinculado a una amenaza para la salud, con repercusiones en todos los ámbitos de la vida de la persona enferma y de sus familiares. Los mismos autores mencionan que es un suceso vital con muchos retos, ocasionando en la mayoría de pacientes una fuerte carga emocional, lo cual deriva en problemas para adaptarse a la enfermedad. Es así que diversos estudios realizados en la población oncológica, sugieren que el 25 a 50\% presenta alteraciones psicológicas, originadas a partir del proceso de enfermedad. Además, a lo largo de todo el proceso, el paciente vive un duelo constante, ya que debe habituarse a las pérdidas que se presenten, como por ejemplo la pérdida de estabilidad, el rol que ejerce dentro de la familia, del mundo social o laboral, entre otras (Cabrera et al., 2017). Asimismo, otros factores pueden desencadenar sentimientos de ansiedad y depresión, como por ejemplo los síntomas físicos, tales como el dolor y cansancio; los cambios en la imagen corporal, afectando la autoestima y confianza en sí mismo; y el miedo a la muerte, al sufrimiento y al dolor (Asociación Americana de Cáncer, 2016).

Siguiendo esta línea, no sólo es una situación personal, sino que, también, involucra al entorno más cercano del paciente, tal como la familia y amigos (Salaverry, 2013). Para Cruzado (2010) el apoyo familiar, de pareja, social y laboral, así como la vulnerabilidad psicológica previa y la historia de cáncer familiar, son determinantes fundamentales del afrontamiento del diagnóstico. En ese sentido, Cabrera et al. (2017) postulan que se hace imprescindible el rol del psicólogo dentro del transcurso de esta enfermedad, resaltando así la importancia de la psicooncología, campo interdisciplinar de la psicología y las ciencia biomédicas cuyos objetivos son la intervención al paciente, la familia y el personal sanitario, la prevención e investigación de la influencia de factores psicológicos importantes en el 
proceso de la enfermedad oncológica y, también, la optimización de la mejora del sistema de atención sanitaria (Cruzado, 2010).

Con respecto a las intervenciones psicológicas más utilizadas y que han tenido resultados favorables en el ámbito oncológico, se destacan la terapia de grupo, la intervención educativa, la consejería psicológica y la psicoterapia de orientación cognitiva conductual (Villoria, Fernández, Padierna y González, 2015).

Dentro del contexto peruano, son pocos los hospitales especializados en cáncer. Particularmente, hasta el año 2002 sólo existía uno ubicado en la ciudad de Lima (LINCECI, 2017). Dicha institución, inaugurada oficialmente en 1939, inicia con actividades orientadas a la prevención y asistencia social contra el cáncer, asistencia especializada en sus consultorios externos y de hospitalización y, finalmente, como centro de estudios sobre la enfermedad oncológica. Hoy en día, es un organismo público descentralizado del sector salud, contando así con autonomía presupuestal, administrativa, financiera y normativa para todas las labores que ejerce, como por ejemplo: la prevención, promoción y atención integral al paciente oncológico, priorizando a las personas con menos posibilidades económicos; el control a nivel nacional de los servicios de salud orientados a las enfermedades oncológicas; y realizar actividades de investigación y docencia propias de la institución (INEN, 2017).

En el transcurso de los años setenta, comienza a surgir un esfuerzo constante por dar a conocer la importancia de la intervención psicológica dentro del contexto de la enfermedad oncológica, la cual está caracterizada por muchos mitos, miedos y creencias asociada a la muerte. Por ello, sin contar con un ambiente propio y destinado para ello, se comienzan a realizar pequeños grupos de apoyo y dinámicas a pacientes con la ayuda de psicólogos invitados a la institución (INEN, 2017). Con el paso del tiempo, se comienza a consolidar el Servicio de Psicología, el cual actualmente está integrado con el Servicio de Psiquiatría, dando lugar a la Unidad de Salud Mental, ubicada en el segundo piso del edificio principal de 
la institución. Hoy en día cuenta con ocho psicólogos y un psiquiatra, teniendo como función principal la atención psicológica y psiquiátrica a los pacientes y familiares de los tres grupos poblacionales dentro del hospital (niños, adolescentes y adultos) y, al mismo tiempo, brindar apoyo al personal asistencial. Asimismo, el servicio de psicología cuenta con el apoyo de aproximadamente veinte internos, los cuales están divididos en dos turnos (mañana y tarde) y rotan por periódos trimestrales por distintas áreas del hospital para ejercer sus funciones asignadas, las cuales serán expuestas líneas más abajo. 


\section{CAPÍTULO II: DESCRIPCIÓN DE LAS ACTIVIDADES Y}

\section{TAREAS REALIZADAS}

Las actividades del programa de internado del servicio de psicología dentro de la institución se derivan de una serie de objetivos propios del área y de funciones que debe realizar el interno, las cuales están alineadas a dichos objetivos.

Los tres objetivos principales son: en primer lugar, proveer de servicios evaluativos, diagnóstico y terapia individual a pacientes y/o familiares por consulta externa; luego, brindar acompañamiento psicológico a pacientes y familiares del hospital; y, finalmente, reforzar conocimientos relacionados a la psicología y psicooncología. Derivadas de los objetivos mencionados, se encuentran las funciones de: evaluación y diagnóstico a través de entrevistas psicológicas y de la aplicación y corrección de pruebas psicológicas; elaborar y aplicar un plan de intervención para los casos asignados, mediante la orientación y consejería psicológica; elaborar y realizar talleres psicológicos en función a las problemáticas presentadas en diferentes áreas del hospital; y, por último, ser partícipe de capacitación constante, asistiendo a cursos de formación y realizando exposiciones de lecturas psicológicas asignadas y del caso clínico final del internado.

A continuación, se detallarán las actividades, las cuales se desprenden de las funciones mencionadas, que se realizaron en las distintas áreas del hospital. Es importante recalcar que varias de las actividades se llevaron a cabo en más de un área.

\subsection{Acompañamiento psicológico a pacientes y familiares}

Esta actividad consistió en ingresar a las habitaciones para entrevistar a las personas, indagando principalmente en los siguientes aspectos: cognitivo, tomando en cuenta la orientación en tiempo, espacio y persona, el lenguaje, el volumen de voz, el tipo de pensamiento y si existe o no consciencia de enfermedad; emocional, incluyendo los signos y síntomas que presenta, así como la disposición y colaboración ante la intervención; y, por 
último, el aspecto familiar, en el cual se colocó el apoyo que posee el paciente por parte de sus seres más cercanos, incluyendo a su cuidador primario. De igual manera, se debía entrevistar a los familiares del paciente para brindarles apoyo emocional y familiar. Al finalizar la entrevista, se debía redactar una Nota Psicológica (ver Apéndice 1) en la historia clínica del paciente, reportando a grandes rasgos los aspectos mencionados, una impresión diagnóstica, las técnicas utilizadas y algunas recomendaciones. Cuando fue necesario y pertinente, se solicitó una interconsulta a psiquiatría, la cual debía ser colocada dentro de la nota. Luego, esta era revisada y firmada por el supervisor(a) de área. Asimismo, dentro de esta actividad, se pudo emplear el uso de algunas pruebas psicológicas para detectar a pacientes que necesiten un mayor y seguimiento apoyo psicológico. Las pruebas que se utilizaron para ello fueron la Escala de Ansiedad y Depresión Hospitalaria (HADS) y el Cuestionario de Afrontamiento al Estrés para Pacientes Oncológicos (CAEPO). No obstante, solo se aplicaron en pocas ocasiones ante la solicitud de los supervisores.

Es importante precisar que dentro de cada área hubieron pequeñas variaciones de esta actividad, las cuales serán mencionadas a continuación:

\subsection{1 Área de cirugía}

Particularmente en el área de cirugía, se trabajó mucho la preparación emocional para la operación a la cual iba a ser sometido el paciente, utilizando en gran medida la psicoeducación, acerca de la enfermedad y los procedimientos médicos, y técnicas de relajación. Muchas veces para los pacientes el solo hecho de estar hospitalizados genera altos niveles de angustia, al ser un ambiente desconocido con rutinas y terminologías con las cuales el paciente no está familiarizado (Benitez, Barceló y Gelves, 2016). Dentro de las técnicas de relajación más empleadas están la respiración diafragmática y la imaginación guiada, ya que son ampliamente utilizadas para disminuir la sintomatología del estrés, ansiedad, depresión y, además, ayudan a disminuir el dolor y la tensión (Larios et al., 2018). 
Dichas técnicas se realizaron en el cuarto del paciente o, si era posible para él, en algún ambiente cercano donde no habían muchas distracciones. Primero, se le psicoeducó acerca de los beneficios de aprenderlas y luego, se procedió a enseñarle cómo llevarlas a cabo, indicando el procedimiento, los tiempos, las repeticiones de cada paso, etc.

\subsection{2 Área de pediatría}

En el área de pediatría, la mecánica de ingresar a las habitaciones fue similar a la de cirugía; sin embargo, el acompañamiento y la intervención psicológica varió en función de la edad del niño. En los más pequeños se hizo de una manera más lúdica, es decir, a través de juegos o dibujos, los cuales solían ser solicitados por ellos mismos. Generalmente, se usó cartas, rompecabezas, juguetes, dibujos en blanco para colorear, mandalas, entre otros. De esta manera, se comenzó a formar la alianza terapéutica, se brindó un espacio para expresar sus sentimientos y temores, se resolvieron dudas y, a lo largo de las sesiones, se fue encontrando la necesidad y sintomatología existente en el niño (Asociación Americana de Cáncer, 2015). Por otro lado, en los niños mayores, se comenzó a trabajar igualmente por medio de juegos; no obstante, luego se pudo ir indagando directamente en los aspectos antes mencionados (cognitivo, emocional y familiar).

Dentro de esta área, luego de intervenir al niño, era fundamental la entrevista al cuidador(a) primario(a), que generalmente era el padre o la madre del niño y estaba presente la mayor parte del tiempo en el hospital. Dentro de la nota que se realizaba en el área de pediatría, se reportó lo encontrado en el niño y en el padre o madre.

\subsection{3 Área de adolescentes}

Al igual que las dos áreas mencionadas líneas arriba, se debía ingresar a cada habitación para intevenir a los adolescentes e indagar en los aspectos cognitivo, emocional y familiar y, posteriormente, redactar una nota psicológica en la historia clínica del paciente. Sin embargo, en muchas ocasiones, como en el área de pediatría, se debió comenzar las intervenciones con 
algunos juegos, dibujos o lecturas, ya que era común que por la edad en la que se encontraban hayan mostrado cierta resistencia a la entrevista. Esto debido a que el adolescente debe lidiar con dos fuentes de estrés: en primer lugar, el propio diagnóstico de cáncer y los tratamientos que conlleva y, en segundo lugar, la misma etapa de adolescencia comprende una mayor vulnerabilidad hacia el estrés, la cual se puede ver intensificada por la enfermedad (Bellver y Verdet, 2015).

\subsubsection{Unidad de Tratamiento Multidisciplinario (UTM), Unidad de Tratamiento Intermedio (UTI) y Unidad de Cuidados Intensivos (UCI)}

Estas tres áreas se encuentra comprendidas dentro de la misma rotación. En las áreas de UTM y UTI, las intervenciones solían ser breves y más directas, ya que los pacientes se encontraban en un estado delicado de salud y las condiciones del ambiente eran estrictamente controladas. Igualmente, se debía indagar en los aspectos ya mencionados líneas arriba. Por otro lado, la entrevista y apoyo dentro del área de UCI se dio en gran medida con los familiares, ya que los pacientes generalmente no se encontraron en condiciones para comunicarse. Igualmente, al finalizar se debía redactar la nota psicólogica para ser firmada por el supervisor de área.

\subsection{Elaboración y realización de talleres psicológicos}

Todos los talleres realizados en el hospital se basaron en la misma estructura (ver Apéndice 2), independientemente del área de rotación. La variación se dio en la temática, que fue cambiando según la población y las problemáticas encontradas en cada área.

Es importante precisar que dentro del área de UTM, UTI y UCI, por las características de la población, no se pudo realizar talleres, es por ello que los internos que rotaron por esos ambientes, realizaron los talleres para un grupo de apoyo llamado "Club de la Mama", enfocado en mujeres que presentaban o habían presentado el diagnóstico de cáncer de mama y contaban con un programa de distintas actividades, como por ejemplo yoga, charlas y los 
talleres mencionados. De igual manera, cuando el personal sanitario lo solicitaba, se realizaron talleres para el personal técnico de enfermería o colaboradores del hospital, principalmente acerca de la importancia de una comunicación asertiva, la empatía y la socialización entre pares.

En líneas generales (a excepción de los talleres del "Club de la Mama", que contaban con horarios establecidos y eran parte del programa), se debía ingresar unas horas antes a todos los cuartos del área invitando a los pacientes o familiares que deseen y puedan asistir, debido a que, en muchos casos, algunos pacientes no podían traslardarse o no contaban con autorización de las enfermeras. Una vez que se contaba con los pacientes interesados y aptos para participar del taller, se les acompañaba al ambiente para dar inicio al mismo. La mayoría de ellos fueron acerca de psicoeducación sobre las emociones, relajación (a través de mindfulness o arteterapia) o compartir experiencias para crear un grupo de apoyo entre los mismos pacientes o familiares. Todos los talleres duraron aproximadamente una hora y media. Como ya se mencionó, todos seguían la misma estructura, pero se detallará uno del área de pediatría dirigido a los padres de familia para ejemplificar la actividad. Dicho taller, con título "Un momento para mí", presentó como objetivo principal brindar un espacio de relajación a través de la imaginación guiada a los padres de los niños hospitalizados, ya que por las características de la enfermedad solían estar internados por periodos largos de tiempo y, por ende, los padres permanecían en el hospital acompañandolos todo el tiempo. Dentro de este taller, la primera actividad consistió en que el equipo de psicología se presente y brinde una breve explicación acerca de lo que implica la relajación, su utilidad y beneficios. Luego, se procedió a entregar un termómetro impreso, el cual tenía señalado en diferentes partes del mismo frases que iban desde "nada tenso" hasta "muy tenso", para que los padres coloreen el nivel en el que se encontraban. De esta manera, el equipo contaba con un primer registro del nivel de tensión de los participantes. Seguido a esto, se dio inicio a la imaginación guiada, 
acompañada de algunos sonidos leves relacionados con la narración y respiraciones. Al finalizar la actividad, se les solicitó que vuelvan a colorear el termómetro para que puedan observar la diferencia entre el estado inicial y el actual. Finalmente, se hizo un intercambio de opiniones con los pacientes y se brindó un espacio para aclarar alguna duda que haya podido surgir a lo largo del taller. Posterior a esto, se les invitó al siguiente taller y se les despidió.

\subsection{Orientación y consejería psicológica por consulta externa}

Esta actividad consistió en brindar apoyo y acompañamiento psicológico, mediante la orientación y consejería a pacientes que ya habían sido dados de alta, en los consultorios del departamento. Para ello, los pacientes debían agendar una cita cada quince días para ser atendidos y brindarles el seguimiento correspondiente, a partir del plan de intervención elaborado. La cita duraba una hora y, luego, se debía redactar una nota psicológica con los aspectos más relevantes de la sesión en la historia clínica del paciente, a través del sistema del hospital, es decir de manera virtual.

\subsection{Exposición de lecturas psicológicas}

El departamento de psicología contaba con una serie de lecturas breves sobre diversas temáticas relacionadas a la psicooncología u oncología. Todos los martes y jueves se realizaban las exposiciones por turnos, es decir un interno por día. Para ello, se debía preparar una presentación con los aspectos más relevantes de la lectura y exponerla a los demás internos y a su supervisor de rotación. Una vez finalizada la exposición, el supervisor y los demás internos daban su opinión acerca del tema expuesto.

\subsection{Supervisión de casos}

Al igual que las exposiciones de lectura, todos los lunes, miércoles y viernes se realizaban supervisiones de casos igualmente por turnos, es decir un interno cada día. Para ello, el interno debía presentar un formato (ver Apéndice 3) incluyendo los datos de filiación del paciente (manteniendo la confidencialidad del nombre e historia clínica), los antecedentes 
médicos de mayor relavancia para contextualizar al resto, un pequeño marco teórico sobre la enfermedad y algún aspecto psicológico trabajado en la entrevista, la descripción general del paciente, la transcripción de la entrevista, una impresión diagnóstica, las técnicas brindadas y, finalmente, el plan de intervención a seguir. Una vez finalizada la exposición, el supervisor(a) de área y los internos opinaban sobre lo trabajado y, además, brindaban sugerencias para realizar el seguimiento del paciente. 


\section{CAPÍTULO III: RESULTADOS DE LA INTERVENCIÓN}

A raíz de las actividades mencionadas, se obtuvieron una serie de resultados y logros. Con respecto al acompañamiento psicológico de los pacientes en los tres grupos poblacionales del hospital (niños, adolescentes y adultos), se pudo aportar en el manejo emocional del proceso de enfermedad, a través de un espacio de escucha y de aclarar dudas y/o preocupaciones que les ocasionaban sentimientos de ansiedad y depresión, así como también a través de las diferentes técnicas y estrategias para afrontar dicha sintomatología. Esto se veía reflejado en los comentarios de los pacientes, como por ejemplo: "Por favor, podría venir mañana?", “conversar me hace bien”, “practiqué la respiración que me enseñó, intento hacerla todos los días”, entre otros. Asimismo, mensualmente se logró intervenir aproximadamente a treinta y cinco pacientes, lo cual supone el cumplimiento de la cantidad establecida por los supervisores. Esta actividad también permitió discriminar entre los casos que necesitaban mayor atención psicológica e incluso, a los que debían ser derivados al departamento de psiquiatría para poder brindarles el mejor tratamiento y servicio. Con esta información, se realizaba un seguimiento más constante. De igual manera, en las entrevistas por consulta externa se hizo seguimiento a cinco casos en distintas etapas a lo largo del año. En este caso, se vio evidenciado el logro de la función en la adherencia de los pacientes a la intervención psicológica, asistiendo puntualmente a sus citas o, en caso no puedan asistir por algún motivo, reprogramando la cita para poder acudir.

Por otro lado, el estar en constante interacción con los pacientes, permitió identificar problemáticas en común para ser resueltas en los talleres psicológicos. En relación a dichos talleres, un indicador que reflejaba el impacto positivo que se obtuvo fue el incremento de pacientes asistentes a los mismos. En un inicio, eran pocos los que se animaban a participar; sin embargo, tras cada taller y sus comentarios positivos, los pacientes o familiares invitaban a otros a participar. Por ejemplo, algunos de los comentarios realizados fueron: “deberían 
hacer más de uno por semana", "me siento más apoyado por el grupo, ellos también atraviesan por algo similar", "es como un tiempo para nosotros", "me gusta asistir porque aprendemos cosas que deberíamos saber, como cosas de la enfermedad". A lo largo de todo el año, entre todas las rotaciones, se realizaron aproximadamente veinticinco talleres.

Es así que las actividades mencionadas brindaron un aporte metodológico, ya que a partir de estar en contacto e interacción con la población oncológica se pudo indagar y formular hipótesis sobre la relación o influencia de ciertas variables con otras, contribuyendo así a iniciar nuevas líneas de investigación o implementar mejoras en las mismas.

En relación a las exposiciones, tanto de lecturas como de supervisiones de casos, se logró una constante capacitación y retroalimentación grupal, ya que todos los días de la semana se recibió información teórica nueva o sugerencias acerca de técnicas o alternativas de abordaje para aplicar con los pacientes. Por ende, ambas actividades aportaron teóricamente en la adquisición de nuevos conocimientos relacionados a la psicooncología y oncología, tanto para los internos como para la institución. 


\section{CONCLUSIONES}

A partir de las actividades ejecutadas y los resultados obtenidos, se concluye lo siguiente:

- El acompañamiento psicológico de los pacientes, hospitalizados o por consulta externa, permitió un mejor manejo de los signos y síntomas derivados del proceso de enfermedad y tratamiento, ya sea a través de la psicoeducación, del espacio de escucha ofrecido o de las técnicas brindadas.

- Los talleres psicológicos permitieron aclarar dudas sobre la enfermedad y las emociones implicadas, disminuyendo así los sentimientos de ansiedad y/o depresión. Además, en algunas ocasiones, sirivieron como un espacio de relajación y conexión con ellos mismos, siendo altamente valorados.

- La realización de talleres, además de ofrecer un espacio de psicoeducación y relajación, promovió la integración y socialización de los pacientes y/o familiares, generando así una red de apoyo entre ellos.

- Las exposiciones de lecturas y supervisiones de caso, promovieron la adquisición de nuevos conocimientos relacionados a la psicooncologia y oncología. De esta manera, se pudo ampliar el repertorio de técnicas e información teórica para ser aplicada con los pacientes y/o familiares. 


\section{RECOMENDACIONES}

A continuación, se plantearán una serie de recomendaciones que se derivan de las actividades y resultados mencionados:

- Se recomienda disponer o contar con un espacio más extenso que pueda dar lugar a un mayor número de consultorios para atender a los pacientes por consulta externa, ya que en muchas ocasiones no había disponibilidad de atenderlos por falta de espacio o entre cada cita existía una gran separación de tiempo.

- Realizar los talleres psicológicos por lo menos dos veces por semana, ya que en ciertas ocasiones por motivos de fuerza mayor se tuvieron que suspender algunos y los pacientes participaban de uno cada dos semanas. Además, eran altamente valorados y acogidos.

- Establecer como un actividad fija y cada cierto tiempo la aplicación y corrección de pruebas psicológicas, principalmente en pacientes hospitalizados, que permitan detectar dificultades en el afrontamiento de la enfermedad, tales como el HADS y el CAEPO, ya que se hizo uso de esta modalidad en pocas ocasiones.

- Incrementar el número de supervisiones de caso al mes por cada interno, ya que esta actividad promueve el intercambio de opiniones, sugerencias de abordaje y/o intervención psicológico y la adquisición de nuevos conocimientos, enriqueciendo así la calidad de las siguientes intervenciones. Debido al número del grupo de internos, muchas veces se realizó solo una supervisión al mes. 


\section{REFERENCIAS}

Asociación Americana de Cáncer (2015). Uso de los servicios de apoyo psicológico.

Recuperado de https://www.cancer.org/es/tratamiento/los-ninos-y-el-cancer/cuandosu-hijo-tiene-cancer/comprension-del-sistema-de-atencion-medica/apoyopsicosocial.html

Asociación Americana de Cáncer (2016). La ansiedad, el miedo y la depresión. Recuperado de https://www.cancer.org/es/tratamiento/tratamientos-y-efectos-secundarios/efectossecundarios-emocionales/ansiedad-miedo-depresion.html

Bellver, A. y Verdet, H. (2015). Adolescencia y cáncer. Psicooncología, 12(1), 141-156. doi: 10.5209/rev_PSIC.2015.v12.n1.48909

Benítez-Agudelo, J., Barceló-Martínez, E. y Gelves-Ospina, M. (2016). Características psicológicas de los pacientes con larga estancia hospitalaria y propuesta de protocolo para su manejo clínico. Cirugía Plástica Iberolatinoamericana, 42(4), 391-398. doi: $10.4321 / \mathrm{S} 0376-78922016000400012$

Cabrera, Y., López González, E ., López Cabrera, E. y Arredondo, C. (2017). La psicología y la oncología: en una unidad imprescindible. Revista Finlay, 7(2), 115-127. Recuperado de http://www.revfinlay.sld.cu/index.php/finlay/article/view/522/1570

Cruzado, J. (2010). Tratamiento psicológico en pacientes con cáncer. Madrid: Síntesis.

Fernández, B., Jorge, B., Sánchez, C. y Bejar, E. (2016). Atención psicológica para pacientes con cáncer y sus familiares: ¿qué nos encontramos en la práctica clínica? Psicooncología, 13(2-3), 191-204. doi: 10.5209/PSIC.54432

Instituto Nacional de Enfermedades Neoplásicas (2014). Casos nuevos de cáncer registrados en el INEN, periodo 2000-2014 (ambos sexos). Recuperado de http://www.inen.sld.pe/portal/documentos/pdf/estadistica/datos_estadisticos/020620 
Instituto Nacional de Enfermedades Neoplásicas (2017). Resultados de la implementación del programa presupuestal de control y prevención en salud mental en el INEN, periodo 2015-2016. Recuperado de http://portal.inen.sld.pe/wp-content/uploads/2018/03/PpRSALUD-MENTAL-RESULTADOS-2015-2016.pdf

Larios, F., González, L., Montes, R., González, F., Morán, A., Solano, P., ..., Daneri, A. (2018). Eficacia de las técnicas de relajación en la disminución de tensión, ansiedad y estrés percibidos por pacientes con cáncer bajo tratamiento de quimioterapia. Gaceta Mexicana de Oncología, 17, 93-99. doi: 10.24875/j.gamo.M18000138

Liga Nacional contra el Cáncer Infantil (2017). Linceci en el hospital oncológico de referencia de toda la región sur de Perú - el IREN Sur. Recuperado de http://linceci.org.pe/linceci-hospital-oncologico-referencia-toda-la-region-sur-peruiren-sur/

Ministerio de Salud del Perú (2014). Principales causas de mortalidad por sexo. Recuperado de http://www.minsa.gob.pe/estadisticas/estadisticas/mortalidad/macros.asp?00 Organización Mundial de la Salud (2018). Nota de prensa: Cáncer. Recuperado de https://www.who.int/es/news-room/fact-sheets/detail/cancer

Salaverry, O. (2013). Psicología en salud. Recuperado de https://books.google.com.pe Villoria, E., Fernández, C., Padierna, E. y González, S. (2015). La intervención psicológica en pacientes oncológicos: una revisión de la literatura (2000-2014). Psicooncología, 12(2-3), 207-236. doi: 10.5209/rev_PSIC.2015.v12.n2-3.51005 


\section{APÉNDICES}




\section{APÉNDICE 1: Modelo de Nota Psicológica}

\section{$\underline{\text { Psicología }}$}

Fecha y hora

Psicólogo(a):

Interno (a):

Se aborda a paciente (mujer/varón) de años con Dx de Se muestra con actitud ante intervención y al momento de la entrevista se encuentra

A nivel cognitivo, se evidencia

Emocionalmente, muestra

A nivel familiar, posee soporte, representado principalmente por

IDX:

Se brinda:

Plan de TTO: 


\section{APÉNDICE 2: Formato de Taller Psicológico}

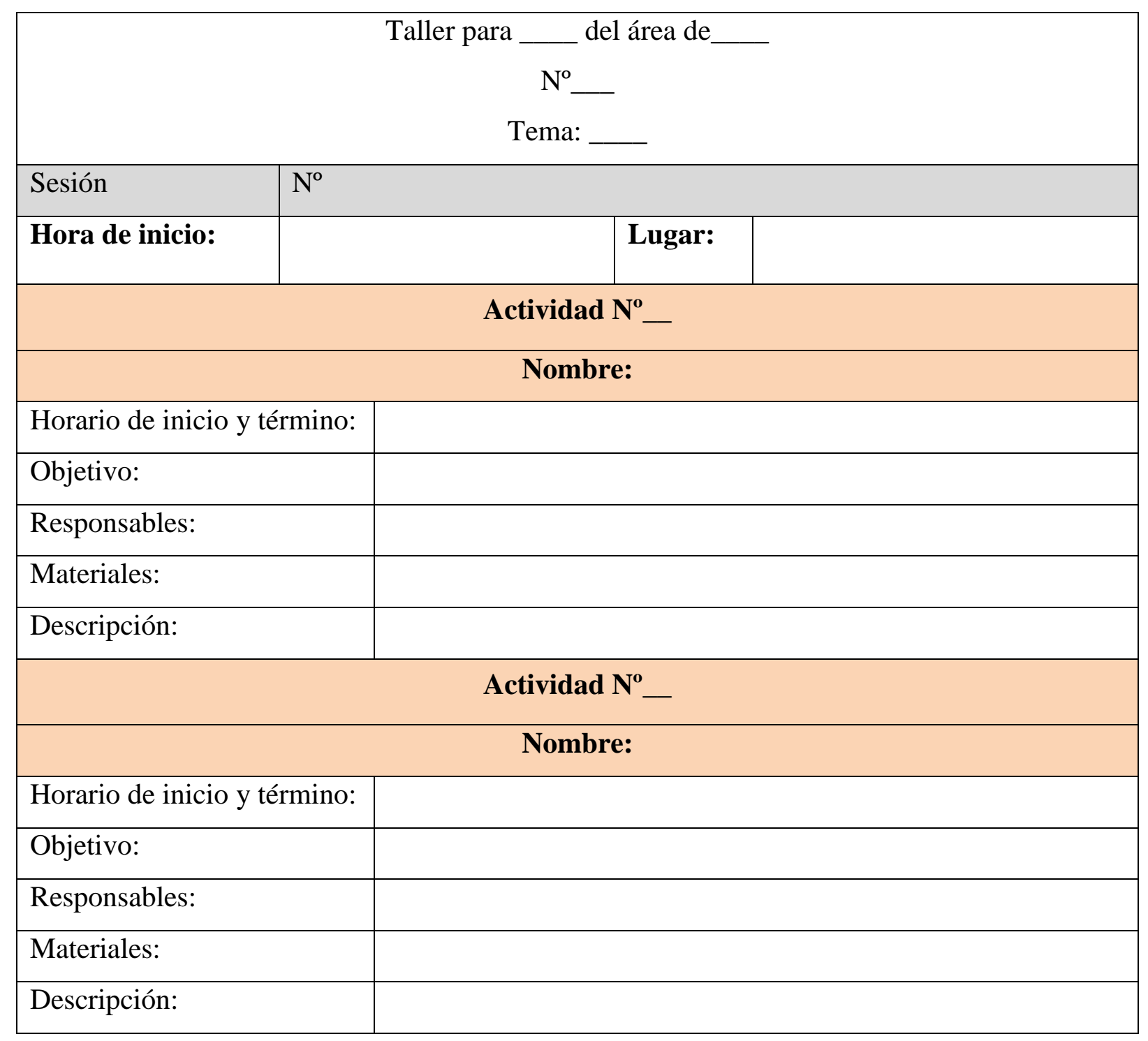




\section{APÉNDICE 3: Modelo de Supervisión de Caso}

\section{Supervisión 1: Caso X}

I. Datos de filiación

- Nombre:

- Edad:

- Lugar de procedencia:

- Hijos:

- Vive con:

- Religión:

- Diagnóstico médico:

- Dpto. Médico:

- Fecha de entrevista:

II. Antecedentes médicos

III. Marco Teórico

IV. Descripción general

V. Transcripción

VI. Impresión diagnóstica

VII. Técnicas brindadas

VIII. Plan de intervención

Referencias 